\title{
Turing computability of the Solution Operator of a higher order modified Camassa-Holm equation
}

\author{
Dianchen $\mathrm{Lu}^{1, \mathrm{a}^{*}}$ and Qiaoqiao Chen ${ }^{1, \mathrm{~b}}$ \\ ${ }^{1}$ Nonlinear Scientific Research Center, Faculty of Science, Jiangsu University, \\ Zhenjiang Jiangsu, 212013, P.R.China \\ aEmail: dclu@ujs.edu.cn, b ${ }^{\mathrm{b}}$ Email: 760450974@qq.com
}

\begin{abstract}
Keywords: Modified Camassa-Holm equation, Solution operator, Turing computability, TTE theory, Duhamel principle
\end{abstract}

Abstract. In this paper, we mainly discuss the Turing computability of the solution operator of a higher order modified Camassa-Holm equation. Firstly, we transform the equation to its integral equation by Duhamel principle. Then applying the TTE theory, we prove that the solution operator of the integral equation is computable in a short interval. Finally, by constructing computable function, we extend the solution from partial internal to the entire space. The result enlarge the application in computing differential equations on digital computers.

\section{Introduction}

Camassa-Holm equation is an important equation. In the shallow water wave

theory, different nonlinear completely integrable partial differential equation can be obtained by different levels of approximation. These equations with soliton solution, that is, they tend to be the determine constant in the infinity of space. Not disappear when they collide with each other and waveform, and speed also have no or only a small change. In addition, they show similar particle scattering behavior.

In this paper, for $m \geq 3$ odd, we will prove that the solution operator of the initial problem(1) is computable by the TTE theory [1-3].

$$
\left\{\begin{array}{l}
\partial_{t} u+\partial_{x}^{m} u+\frac{1}{2} \partial_{x}\left(u^{2}\right)+\left(1-\partial_{x}^{2}\right)^{-1} \partial_{x}\left[u^{2}+\frac{1}{2}\left(\partial_{x} u\right)^{2}\right]=0 \\
u(x, 0)=\varphi(x), t \in R, x \in R .
\end{array}\right.
$$

Erika A.Olson show in [4] that the periodic initial value problem for Eq.(1) is well posed.

At present, the computability of solutions of the nonlinear evolution equations

have become an important topic to the workers of physics and mathematics [5-6]. Researching boundedness and computability of the solutions of the nonlinear equations will offer effective tools for the application of equations, enrich theoretical foundation of computer science and promote the development of computer software.

The structure of the article is that: In part 2, we introduce some basic definitions and lemmas, which are relevant to the proof of part3; In part 3, we prove the main theorem of the paper mainly.

\section{Preliminaries}

Definition $\mathbf{1}^{[4]}$ For any $\forall s, b \in R, X^{s, b}$ denotes the completion of the Schwartz space $S\left(R^{2}\right)$ with respect to the norm

$$
\|u\|_{s, b}^{\prime}=\left(\int_{R} \int_{R}(1+|\xi|)^{2 s}\left(1+\left|\tau-\xi^{m}\right|\right)^{2 b}|\hat{u}(\xi, \tau)|^{2} d \xi d \tau\right)^{\frac{1}{2}} .
$$


Definition $2^{[4]}$ In the sequel, let $\psi=\psi(t) \in C_{0}^{\infty}(-1,1)$ be a cut-off function with $0 \leq \psi \leq 1$ and

$$
\psi(t) \equiv 1 \text { for }|t|<\frac{1}{2} \text {. }
$$

Definition $3^{[4]}$ For $0<\delta<1$, define $\psi_{\delta}(t)=\psi\left(\frac{t}{\delta}\right), T_{\delta} u=\psi_{\delta} T\left(\psi_{\delta} u\right)$.

\section{Lemma $1^{[1]}$ (type conversion)}

Let $\delta_{i}: \subseteq \sum^{\omega} \rightarrow X_{i}$ be a representation of the set $X_{i}(0 \leq i \leq k)$,

$f_{i}: \subseteq X_{1} \times \cdots \times X_{k} \rightarrow X_{0} \quad$, and define $L\left(x_{1}, \cdots, x_{k-1}\right)\left(x_{k}\right):=f\left(x_{1}, \cdots, x_{k}\right)$, then if $f$ is $\left(\delta_{1}, \cdots, \delta_{k}, \delta_{0}\right)$-computable if and only if $L$ is $\left(\delta_{1}, \cdots, \delta_{k-1},\left[\delta_{k} \rightarrow \delta_{0}\right]\right)$-computable.

Lemma $2^{[1]}$ Let $\gamma: \subseteq Y \rightarrow M$ and $\gamma^{\prime}: \subseteq Y \rightarrow M^{\prime}$ are two representations, $v_{N}$ is admissible representation of $N$. Then we have the following propositions:

1) If $f: \subseteq M \rightarrow M^{\prime}$ is $\left(\gamma, \gamma^{\prime}\right)$ - computable, then $f^{\prime}: \subseteq N \times M^{\prime} \times M \rightarrow M^{\prime}$ is $\left(v_{N}, \gamma^{\prime}, \gamma, \gamma^{\prime}\right)$ - computable.

We define a function $g^{\prime}: \subseteq N \times M \rightarrow M^{\prime}$ as follow:

$$
g^{\prime}(0, x)=f(x), g^{\prime}(n+1, x)=f^{\prime}\left(n, g^{\prime}(n, x), x\right),
$$

where $x \in M, n \in N$, then $g^{\prime}$ is $\left(v_{N}, \gamma, \gamma^{\prime}\right)$ - computable.

2) Assuming that $h: \subseteq M \rightarrow M$ is $(\gamma, \gamma)$ - computable, Define a function

$$
\begin{gathered}
H: \subseteq N \times M \rightarrow M \\
H(0, x)=x, H(n+1, x)=h \circ H(n, x)=h^{n+1}(x) .
\end{gathered}
$$

So, the function $H$ is $\left(v_{N}, \gamma, \gamma\right)$ - computable.

Lemma $\quad 3^{[4]} \quad$ Given $\quad s \in\left(\frac{1}{2} \cdot \frac{m^{2}-3 m+1}{m^{2}-3 m+2}, \frac{1}{2}\right)$, there exists $\beta>0$ such that if $b \in\left(\frac{1}{2}, \frac{1}{2}+\beta\right], b^{\prime} \in\left[\frac{1}{2}-\beta, \frac{1}{2}\right)$, and $b+b^{\prime} \leq 1$, then there exists $c>0$ such that for any $f, g \in X^{s, b^{\prime}},\left\|w_{f g}\right\|_{s, b-1}^{\prime} \leq c\|f\|_{s, b^{\prime}}^{\prime}\|g\|_{s, b^{\prime}}$, where $w_{f g}$ satisfies $\widehat{w}_{f g}(\xi, \tau) \square\left(\xi+\frac{2 \xi}{1+\xi^{2}}\right) f * g(\xi, \tau)+\frac{\xi}{1+\xi^{2}} \partial_{x} f * \partial_{x} g(\xi, \tau)$.

\section{Main Result}

From the problem (1), we establish a nonlinear map $K_{R}: H^{s} \rightarrow C\left(R ; H^{s}(R)\right)$,

which translate the initial data $\varphi$ to the solution $u$. The map $K_{R}$ is the solution operator of the initial problem.

Theorem 3.1 For any $t \in R$, when $\frac{1}{2} \cdot \frac{m^{2}-3 m+1}{m^{2}-3 m+2}<s<\frac{1}{2}$, the solution operator $K_{R}: H^{s} \rightarrow C\left(R ; H^{s}(R)\right)$ is $\left(\delta_{H^{s}},\left[\rho \rightarrow \delta_{H^{s}}\right]\right)$-computable, where $m \geq 3$ odd.

Proof Defining $w=\frac{1}{2} \partial_{x}\left(u^{2}\right)+\left(1-\partial_{x}^{2}\right)^{-1} \partial_{x}\left[u^{2}+\frac{1}{2}\left(\partial_{x} u\right)^{2}\right]$, We can get Eq.(1) equivalent integral equation by Duhamel principle:

$$
u(x, t)=W(t) \varphi(x)-\int_{0}^{t} W(t-\tau) w(x, \tau) d \tau .
$$


where $W(t) \varphi(x):=\int_{R} \hat{\varphi}(\xi) e^{i\left(\xi x+\xi^{m} t\right)} d \xi$.

$$
\begin{aligned}
& \text { For } \varphi \in H^{s}\left(\frac{1}{2} \cdot \frac{m^{2}-3 m+1}{m^{2}-3 m+2}<s<\frac{1}{2}\right) \text {, define operator } \\
& S(u, \varphi, t)=\psi(t) W(t) \varphi(x)-\psi_{\delta}(t) \int_{0}^{t} W(t-\tau) w(x, \tau) d \tau, \\
& \bar{S}(u, \varphi)(t):=S(u, \varphi, t) .
\end{aligned}
$$

According the Lemma 3.2 in [1], it is easy to prove $S$ is $\left(\left[\rho \rightarrow \delta_{s}\right], \delta_{s}, \rho, \delta_{s}\right)$-computable.

By lemma $1, \bar{S}$ is $\left(\left[\rho \rightarrow \delta_{s}\right], \delta_{s},\left[\rho \rightarrow \delta_{s}\right]\right)$-computable.

Define function $v: S(R) \times N \rightarrow C(R ; S(R))$

$$
v(\varphi, 0)=\bar{S}(0, \varphi), v(\varphi, j+1)=\bar{S}(v(\varphi, j), \varphi) .
$$

The function $v$ is defined by primitive recursion from computable functions. By Lemma $2 v$ is $\left(\delta_{s}, v_{N},\left[\rho \rightarrow \delta_{s}\right]\right)$-computable.

Let $\omega(x, t)=u\left(x, t_{0}+t\right)$, where $t \in[0, T], t_{0} \geq 0$.

If $u\left(x, t_{0}\right)=\mu(x)$, then

$$
\left\{\begin{array}{l}
\partial_{t} \omega+\partial_{x}^{m} \omega+\frac{1}{2} \partial_{x}\left(\omega^{2}\right)+\left(1-\partial_{x}^{2}\right)^{-1} \partial_{x}\left[\omega^{2}+\frac{1}{2}\left(\partial_{x} \omega\right)^{2}\right]=0 \\
\omega(x, 0)=\mu(x), t \in R, x \in R .
\end{array}\right.
$$

We assume that the initial value $\mu \in H^{s}(R)$ is given by a $\tilde{\delta}_{H^{s}}$-name, i.e., $\quad p=\left\langle p_{0}, p_{1}, \cdots\right\rangle$ which is obtained by $\delta_{s c}\left(p_{i}\right)=\mu_{i}$ and $\left\|\mu_{n}-\mu\right\|_{s} \leq 2^{-n-2}$. For $\forall k \in N$, there exist appropriate computable $n_{k}$ satisfying $\left\|\mu_{n_{k}}-\mu\right\|_{H^{s}} \leq 2^{-n_{k}-2} \leq 2^{-k-2}$.

Define

$$
\omega_{n}^{0}:=\bar{S}\left(0, \mu_{n}\right), \omega_{n}^{j+1}:=\bar{S}\left(\omega_{n}^{j}, \mu_{n}\right) .
$$

It is easy to prove $\omega_{n}^{j} \rightarrow \omega_{n} \quad(j \rightarrow \infty)$, there $\omega_{n}$ satisfies the following integral equation:

$$
\omega_{n}(t)=\bar{S}\left(\omega_{n}, \mu_{n}\right)=\psi(t) W(t) \mu_{n}(x)-\psi_{\delta}(t) \int_{0}^{t} W(t-\tau) w(x, \tau) d \tau,
$$

where $w=\frac{1}{2} \partial_{x}\left(\omega_{n}^{2}\right)+\left(1-\partial_{x}^{2}\right)^{-1} \partial_{x}\left[\omega_{n}^{2}+\frac{1}{2}\left(\partial_{x} \omega_{n}\right)^{2}\right]$.

Since $\omega_{n}^{j} \rightarrow \omega_{n} \quad(j \rightarrow \infty)$, we can select suitable integer $n_{k}, j_{k}$ to constrct a sequence $\left\{\omega_{n_{k}}^{j_{k}}\right\}_{k \in N}$, satisfying $\left\|\omega_{n_{k}}^{j_{k}}-\omega_{n_{k}}\right\|_{s} \leq 2^{-k-1}$.

In the following, we prove $\left\{\omega_{n_{k}}^{j_{k}}\right\}_{k \in N}$ fastly converges to $\omega$.

By the Lemma 3, we obtain

$$
\begin{aligned}
& \left\|\omega_{n_{k}}-\omega\right\|_{s, b}^{\prime}=\left\|\psi(t) W(t)\left(\mu_{n_{k}}(x)-\mu(x)\right)-\psi_{\delta}(t) \int_{0}^{t} W(t-\tau)\left[w_{\omega_{n_{k}}}(x, \tau)-w_{\omega}(x, \tau)\right] d \tau\right\|_{s, b} \\
& \leq\left\|\psi(t) W(t)\left(\mu_{n_{k}}(x)-\mu(x)\right)\right\|_{s, b}^{\prime}+\left\|\psi_{\delta}(t) \int_{0}^{t} W(t-\tau)\left[w_{\omega_{n_{k}}}(x, \tau)-w_{\omega}(x, \tau)\right] d \tau\right\|_{s, b} \\
& \leq\left\|\psi(t) W(t)\left(\mu_{n_{k}}(x)-\mu(x)\right)\right\|_{s, b}^{\prime}+\left\|\psi_{\delta}(t) \int_{0}^{t} W(t-\tau) w_{f g}(x, \tau) d \tau\right\|_{s, b}^{\prime} \\
& \leq C_{1}\left\|\mu_{n_{k}}(x)-\mu(x)\right\|_{H^{s}}+C \delta^{\frac{1}{2}-b}\left\|\omega_{n_{k}}+\omega\right\|_{s, b}^{\prime}\left\|\omega_{n_{k}}-\omega\right\|_{s, b}^{\prime}
\end{aligned}
$$


$\leq C_{1} 2^{-k-2}+2 r C \delta^{\frac{1}{2}-b}\left\|\omega_{n_{k}}-\omega\right\|_{s, b}^{\prime}$, where $f=\omega_{n_{k}}+\omega, g=\omega_{n_{k}}-\omega$.

Choosing sufficient small $\delta$ such that $\frac{C_{1}}{1-2 r C \delta^{\frac{1}{2}-b}}<2$, then $\left\|\omega_{n_{k}}-\omega\right\|_{s, b}^{\prime} \leq 2^{-k-1}$.

Thus $\left\|\omega_{n_{k}}^{j_{k}}-\omega\right\|_{s, b}^{\prime} \leq\left\|\omega_{n_{k}}^{j_{k}}-\omega_{n_{k}}\right\|_{s, b}^{\prime}+\left\|\omega_{n_{k}}-\omega\right\|_{s, b}^{\prime} \leq 2^{-k-1}+2^{-k-1} \leq 2^{-k}$.

Then we have proved $\left\{\omega_{n_{k}}^{j_{k}}\right\}_{k \in N}$ fastly converges to $\omega$ and $\omega$ is computable.

We known $\left\{\omega_{n_{k}}^{j_{k}}\right\}_{k \in N}$ is computable sequence, if $\delta_{S}\left(q_{k}\right)=\omega_{n_{k}}^{j_{k}}(t)$, then $\tilde{\delta}_{H^{s}}\left\langle q_{0}, q_{1}, \ldots\right\rangle=\omega(t)$, i.e., $\left\langle q_{0}, q_{1}, \ldots\right\rangle$ is the $\tilde{\delta}_{H^{s}}$-name of $\omega(t)$. Hence the solution $\omega$ of the initial problem (3) is computable on $t \in[0, T]$, that is solution operator map $S$ is computable.

Define a $\left(\rho, \tilde{\delta}_{H^{s}}, \rho, \tilde{\delta}_{H^{s}}\right)$-computable map $P:\left(t_{0}, \mu, t\right) \rightarrow u(t), t \in\left[t_{0}, t_{0}+T\right]$, where $\omega\left(t_{0}\right)=\mu, \omega(t)$ is the solution of the initial problem (1) on $t \in\left[t_{0}, t_{0}+T\right]$.

Then we prove the solution $u(n \cdot T)$ is computable.

Define function $H: H(\varphi, n)=u(n T)$

$$
H(\varphi, 0)=\varphi, H(\varphi, n+1)=P(n T, H(\varphi, n),(n+1) T) .
$$

Then $H$ is computable since $H$ is derived by primitive recursion from computable function $P$.

In the end, we prove $u(t)$ is computable. let $n \cdot T \leq t \leq(n+1) \cdot T$, we first compute $u(n \cdot T)$, then compute $P(n T, u(n T), t)$, so $u(t)=P(n T, u(n T), t)$ is computable.

In this way, we have get the computable solution on $t \in R$. For $\forall t \in R, \frac{1}{2} \cdot \frac{m^{2}-3 m+1}{m^{2}-3 m+2}<s<\frac{1}{2}$, the solution operator $K_{R}: H^{s} \rightarrow C\left(R ; H^{s}(R)\right)$ of the higher order modified Camassa-Holm equation $(1)$ is $\left(\delta_{s},\left[\rho \rightarrow \delta_{s}\right]\right)$-computable.

\section{Summary and Outlook}

The paper study computability of the solution operator of the higher order modified Camassa-Holm equation. On the basis of computablity theory, whether problem can be implemented on computer is an important problem. Computational complexity theory just can be used to solve the problem. The topic we will study in the future.

\section{Acknowledgements}

The work was supported by the National Nature Science Foundation of China (61070231) and the Natural Science Foundation of Jiangsu Province (BK20140525).

\section{References}

[1] K.Weihrauch, N.Zhong. Computing the solution of the Korteweg-de Vries equation with arbitrary precision on Turing machines. Theoret.Comput.Sci.. 2005.332 (1-3): 337-366.

[2] Ning Zhong, Klaus Weihrauch, Computability theory of generalized functions [J]. Assoc. for Computing Mathinery 50 (4) (2003)469-505.

[3] N. Zhong. Computability structure of the Sobolev spaces and its applications. Theoretical Computer Science. 1999 (219): 487-510.

[4] Erika A.Olson. Well posedness for a higher order modified Camassa-Holm equation.J. Differential Equations 2009 (246) :4154 - 4172. 
[5] Dianchen Lu, Qingyan Wang. Computing the solution of the m-Korteweg-deVries equation on Turing machines, Electronic Notes in Theoretical Computer Science, 2008(202):219-236.

[6] Dianchen Lu, Jiaxin Guo. Computable analysis of the solution of the Nonlinear Kawahara equation.IJCSET.Vol 2(2012),Issue 4,1059-1064. 